\title{
Las dos caras de la moneda Relatos de la Posguerra Civil en Costa Rica ${ }^{1}$
}

\author{
The two sides of the coin. Stories of the Civil Post-War in Costa Rica
}

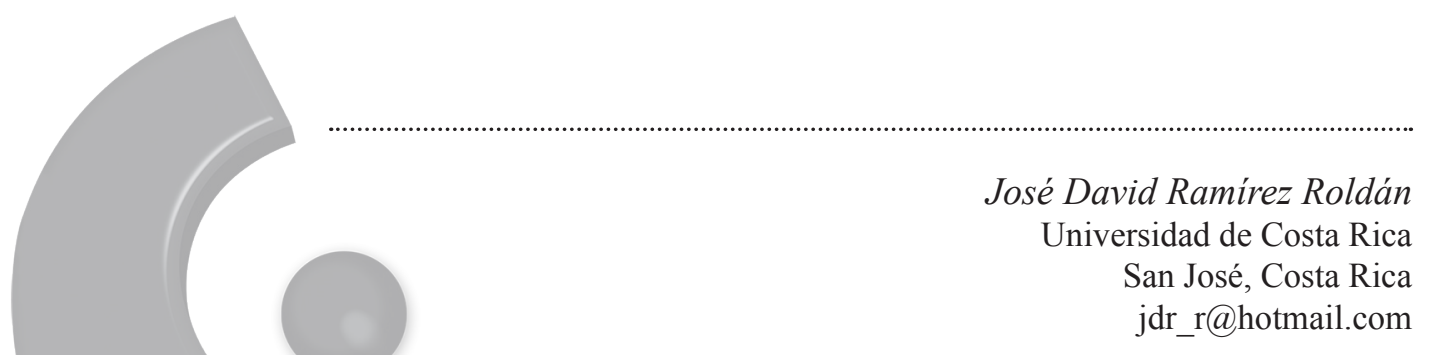

Recibido: 12/03/2017 Aceptado: 16/06/2017

Resumen: La presente investigación pretende comprender las experiencias vivenciales de dos testigos varones entorno a eventos ocurridos a raíz de la Guerra y Posguerra Civil de 1948 en Costa Rica. Cabe señalar, que el hecho en cuestión los dejó marcados, de una u otra manera, para el resto de la vida en su desarrollo personal. El primero, quien vivió dicha experiencia bélica en su infancia como hijo de un dirigente comunista asesinado en el Codo del Diablo a inicios de la posguerra a manos del Estado de Costa Rica; y el segundo, atestiguó dichos eventos durante su época de adolescente, en la cual fue un combatiente del bando "figuerista" durante la guerra. Dichos relatos fueron recolectados por medio de dos entrevistas semiestructuradas adecuadas para cada caso en particular, con una duración cercana entre media y una hora cada una, las cuales fueron realizadas en el primer semestre del año 2015. Asimismo, el trabajo ofrece un balance del material teórico-metodológico y contextual que se consideró adecuado como una vía de análisis histórico con respecto a las fuentes testimoniales utilizadas.

1 La investigación fue producida en el primer semestre del año 2015. 
Revista Universidad en Diálogo • Vol. 7, N. 1, Enero-Junio 2017, pp. 89-99

ISSN 2215-2849 • EISSN: 2215-4752

DOI: http://dx.doi.org/10.15359/udre.7-1.5

Palabras clave: historia oral, Guerra y Posguerra Civil de Costa Rica, mártires del Codo del Diablo, excombatientes adolescentes.

\begin{abstract}
The present paper aims to understand the life experiences of two male witnesses about events that occurred in the aftermath of the 1948 Civil War and Post-War in Costa Rica. It should be noted that the fact in question left them marked, in one way or another, for the rest of life in their personal development. The fisrt one, who lived the experience of war in his childhood as the son of a Communist leader killed in the Codo del Diablo early in the postwar period at the hands of the Costa Rican State; and the second, witnessed these events during his adolescent time, in which he was a combatant of the "figuerista" side during the war. These stories were collected through two semi-structured interviews that were adequate for each particular case, with duration between half and one hour each, which were carried out in the first half of 2015. The research also offers a theoretical-methodological and contextual studies balance that was considered adequate as a way of historical analysis with respect to the testimonial sources used.
\end{abstract}

Keywords: oral history, War and Post-Civil War in Costa Rica, martyrs of Codo del Diablo, ex-combatants adolescents.

Para Elizet Payne Iglesias: amiga, maestra y modelo a seguir.

For Sophia Joy Cox-wright: who always gives me a happy day just with a greeting.

\title{
Introducción
}

En esta investigación se pretende dar a conocer y analizar las experiencias vividas de dos participantes varones quienes fueron afectados directamente durante la Guerra y Posguerra Civil de 1948 en Costa Rica. Uno de ellos, la vivió de niño como hijo de un dirigente comunista asesinado en el Codo del Diablo a inicios de la posguerra; el otro, durante su época de adolescente, fue un combatiente del bando "figuerista" durante la guerra. El conocimiento de esa experiencia fue recolectado por medio de dos entrevistas semiestructuradas, con una duración entre media hora y una hora cada una, realizadas en el primer semestre del año 2015. Empero, las fuentes orales utilizadas fueron estudiadas desde una adecuada revisión del material teórico y contextual, por ende, se pretende primero, mostrar la riqueza del testimonio de estas dos personas de la tercera edad, vistas como una fuente histórica (primaria) para conocer ese pasado vivencial no tan lejano y contemporáneo; en segundo lugar, comprender por medio de las reminiscencias sus experiencias individuales en torno a los hechos, 
consultando sus transiciones personales durante la Guerra y Posguerra Civil, además de sus posibles afinidades políticas posteriores.

Ante aquellos relatos de juventud que, se enmarcan en la relación existente entre historia y memoria, me pregunto: ¿de qué manera se constituye el recuerdo en los vínculos tanto materiales como emocionales, existentes entre la vida cotidiana, el proceso de transición nacional de la Posguerra Civil y las características individuales de dos entrevistados de la tercera edad?

La especialista en historia de España en el siglo XX y memoria histórica, Josefina Cuesta Bustillo, propone una perspectiva que concuerda y clarifica la intención final de cómo podemos interpretar el pasado vivencial, desde la historia y la memoria, expresando que:

Entre el saber cientifico de los hechos pasados, la historia entendida como un saber acumulativo con sus improntas de exhaustividad, de rigor, de control de los testimonios, de una parte; y por otra parte, la memoria de estos hechos pasados cultivada por los contemporáneos y sus descendientes. (Cuesta Bustillo, 1991, p. 3-4)

El interés de este trabajo no es utilizar la fuente oral como un insumo para conocer o interpretar las expresiones de la estructura social. Por ende, no se instrumentalizará la fuente oral en confrontación con la fuente escrita, con el fin, de evadir cualquier riesgo de "...no mal interpretar la especificidad del testimonio y de subestimar su riqueza” (Acuña, 1989, p. 163).

Nos acercaremos, cuidadosamente, a analizar la historia y la memoria presentes en los relatos contenidos en las dos entrevistas realizadas, buscando una "...historia de vida que permita traducir la cotidianidad en palabras, gestos, símbolos, anécdotas, relatos y constituya una expresión de la permanente interacción entre historia personal e historia social" (Puyana y Barreto, 1994, p. 185).

Es formidable la distinción que realiza el historiador costarricense Víctor Hugo Acuña (1989, p. 237), al afirmar que "conviene indicar la diferencia que existe entre una historia de vida temática que abarca un período o un aspecto de la persona entrevistada y la historia de vida total que engloba las distintas dimensiones de la vida de un individuo".

Nuestro caso es una aproximación a la historia de vida temática, es por ello, que los testimonios contados son recuerdos matizados con nostalgia de una Costa Rica dividida por la guerra del 48. 
No interesará constatar la veracidad de los hechos que vayan apareciendo en los testimonios. Por ende, según la antropóloga Mayra Zapparoli (2003, p. 2), hay que entender los planteamientos que hay entre la distinción de la historia como disciplina científica y la construcción social del recuerdo, o más complicado aún, precisar sus relaciones. La obra The voice of the past. Oral History (Thompson, 1978) fue un estudio preliminar sobre esta distinción conceptual, pero sin profundizar en los mismos. Casi una década después, el destacado historiador británico Eric Hobsbawm (1989, p. 205) ofreció un emergente análisis para tratar las fuentes orales y su distinción con las escritas, haciendo la diferencia entre la historia vivida y la historia como escritura. Además, también señaló en su libro La era del Imperio, que "cuando los historiadores intentan estudiar un periodo del cual quedan testigos supervivientes se enfrentan, y en el mejor de los casos se complementan, dos conceptos diferentes de la historia: el erudito y el existencial, los archivos y la memoria personal".

Al iniciar el siglo XXI, para el caso costarricense aparece un espectacular trabajo inscrito en esta misma corriente historiográfica, la cual hizo eco en la distinción entre memoria e historia, el trabajo se dividió en dos tomos con el nombre de Niños y niñas del 48 escriben (Muñoz, 2003). El trabajo toma en cuenta temáticas muy afines al interés de mí investigación, con la excepción, de que las fuentes orales presente en el libro fueron escritas por los adultos que habían sido niños y niñas durante la Guerra de 1948, y en mi caso, se realizaron entrevistas personales.

Interesante sería, estudiar la elección de preferir el silencio, vista como una barrera entre un sentimiento que trata de ocultar algún evento traumático, en otras palabras, algo que no se debe recordar. O sea, pensar en un concepto de trauma que tiene especial relevancia para nuestra comprensión de la herencia de las catástrofes colectivas, con respecto a lo que se ha dicho antes sobre las experiencias traumáticas y los procesos de la memoria, como lo ha señalo, el historiador alemán, Wolf Kansteiner (Zapparoli, 2003, p. 2). Dicho fenómeno, estuvo presente durante muchos años en la vida de uno de nuestros entrevistados, Federico Picado Odio (hijo de Federico Picado Sáenz, quien fue un mártir del Codo del Diablo), quien expresó que durante muchos años, según lo que me ha contado fuera de la grabación en otras ocasiones, guardó silencio por el malestar que causaba recordar el asesinato de su padre, y que en tiempos más recientes, ha vuelto hablar del tema. Para fines de esta investigación, no se consideró importante indagar en reflexiones teóricas sobre el silencio o el olvido que puede causar un hecho traumático, pero sí considero, esencial, tratar la 
expresión del recuerdo que es matizada por el pasar del tiempo, donde el sentimiento de añoranza se albergan en las referencias del pasado; en este caso, en torno a una guerra civil. El silencio, el pesar, la exaltación y la emotividad, son elementos claves para el estudio de ese vínculo, entre historia y memoria. Por ello, hay que tener en consideración que "...junto a los recuerdos puede surgir la figura de un mito, un país sin ejército o un personaje ideal, sea el Dr. Rafael Ángel Calderón, José Figueres Ferrer, o tantos otros..." (Zapparoli, 2003, p. 2).

Los marcos sociales de la memoria, una terminología del sociólogo francés, Maurice Halbwachs, proponen la existencia de un carácter selectivo en los recuerdos, sea la memoria individual o colectiva, ya que puede “...sufrir los embates del tiempo, que no tiene la capacidad de recordarlo todo, y tal vez lo más importante..." (Zapparoli, 2003, p. 3). La influencia del presente en cómo se visualiza el pasado desde el lugar y tiempo donde se recuerda puede afectar la narración. Hay que tener claro, que todo relato está dentro de un espacio geográfico que se tiene que valorar en los estudios de la memoria.

\section{Desarrollo}

\section{Datos personales, geografía de los relatos, familia y cotidianidad modificada}

Lisímaco Elmer Chavarría Trejos (comunicación personal, mayo 29, 2015) es un adulto mayor de 83 años, actualmente vive en Ciudad Colón, tuvo un papel destacado en la historia política contemporánea de Costa Rica, siendo hoy en día un reconocido excombatiente, quien luchó en San Isidro de El General a los 16 años durante la Guerra Civil de 1948 en el bando "figuerista" contra los llamados "Caldero-comunistas", y durante la Posguerra Civil en 1955, la histórica hacienda Santa Rosa de Guanacaste volvió a convertirse en el epicentro de otra guerra, ya que un grupo de rebeldes trataron invadir Costa Rica desde Nicaragua. Don Lisímaco es oriundo de Santa Ana, sus progenitores son Abelardo Chavarría Jiménez, quien era tinterillo, y Elena Trejos Mora, ama de casa, quienes se separaron cuando su hijo tenía 12 o 13 años. Fue descendiente de una familia pobre, prolifera y muy trabajadora, asimismo, le gustaba “... andar buscando trabajos para ganar comida", lo cual lo hizo moverse en varios lugares, hasta llegar a San Isidro de General que fue donde lo “...agarró la revolución”. Concluyó sus estudios superiores, en la Universidad de Costa Rica y en centros de educación superior estadunidenses. Algo muy importante, es cómo lo 
afecto el evento de una Guerra Civil siendo adolescente, ya que personalmente cree que lo convirtió en un hombre adulto valiente; o como diría él: “... los valientes de un lado se matan contra los valientes del otro lado...", de paso, señalándome, que es una frase de su libro Las dos batallas en San Isidro del General (Chavarría, 2014).

Aunque los sentimientos de la posguerra los describe inmersos en un ambiente de confusión, también me afirma que había optimismo por un cambio venidero, que posteriormente se convertiría en hechos de prosperidad económica para muchos sectores de la población, en especial, para el campesinado, ya que después de la nacionalización de la banca, “... apareció el Consejo de la Producción...que compraban los granos a los campesinos...", beneficiándolos bastante, recordando que "...se llegaban con un rollillo de billetes... a comprar" lo que necesitaban. Así, aparecen dos elementos primordiales en la narración, el primero de carácter metafórico, en tanto la vida misma se vive como una guerra, y otro más concreto, con lo que respecta a que la guerra trajo posteriormente en la cotidianidad, interrelacionándose las representaciones y los sentimientos de las personas al hablar de sus vivencias durante la Guerra Civil costarricense. Lo anterior, queda bien retratado con la perspectiva de la socióloga e historiadora, Isabel Gamboa Barboza (2010), quien especifica que se puede entender “...guerra y pobreza al menos en dos sentidos; uno metafórico, en tanto la vida misma se vive como una guerra, y otro concreto, por cuanto la guerra trajo para ellas y ellos vivencias y consecuencias, materiales y emocionales...".

Asimismo, esta interpretación sociológica calza con las descripciones que nos ofrece don Lisímaco, al hablar de cómo en “...esas épocas corrían los billetes, ánimo de esperanza, de futuro...” y sus propios impulsos por “... estudiar por correspondencia con la Castro Carazo, a ver dónde...ganaba un buen sueldo..." para mejorar su situación económica.

Por otro lado, Federico Picado Odio (comunicación personal, junio 20, 2015) es un adulto mayor de 79 años, vecino de Zapote, y como he dicho anteriormente, es hijo de Federico Picado Sáenz, dirigente del Partido Vanguardia Popular de la provincia de Limón, quien el 19 de diciembre de 1948 junto con Octavio Saénz, Lucio Ibarra, Tobías Vaglio, Narciso Sotomayor y Álvaro Aguilar, todos en su mayoría figuras líderes de orientación comunista, que fueron asesinados a manos del Estado costarricense. Así, utilizando las palabras del historiador costarricense Víctor Hugo Acuña, se podría decir que: 
"...fueron ejecutados a mansalva por militares del gobierno de facto de la Junta Fundadora de la Segunda República en un lugar de la vía férrea San José-Limón llamado el Codo del Diablo, cuando por órdenes superiores, eran trasladados en un motocar del cuartel de Limón a la Penitenciaría Central de San José...El crimen se inscribe dentro de las acciones de represión y persecución arbitraria de los vencedores de la guerra civil de 1948 en contra de quienes ellos llamaron los “caldero-comunistas". (Acuña, 2014)

$\mathrm{Al}$ ocurrir el asesinato de su padre, nuestro entrevistado tenía 12 años (F. Picado, comunicación personal, junio 20, 2015), además de una madre viuda con problemas de visión y un hermano pequeño de 6 años, quienes terminaría cuidando. De esta forma, toda su vida cotidiana cambió radicalmente, iniciándose en el trabajo desde muy pequeño para poder mantener económicamente a su familia, pero sin dejar de estudiar en el colegio, de hecho, logró estudiar en la Universidad de Costa Rica, primero Derecho y luego Ciencias Económicas, pero laborar y estudiar con los difíciles horarios de la universidad terminó impidiendo continuar sus estudios. O cómo diría él mismo, con respecto a la causa de la pérdida de su infancia a los doce años: “...cuando mi padre tuvo esa experiencia o tuvimos esa experiencia todos, ahi yo tuve que hacerle frente a todo.... No solo a lo personal...sino, a lo que era mi familita entonces...".

Los espacios geográficos donde se movilizó Picado fueron principalmente en la Ciudad de Cartago, su ciudad de nacimiento, y desde su segundo año de edad en adelante hasta los seis años, estuvo viviendo en Turrialba, después de los siete años, se trasladaron a Limón, donde permaneció hasta los 17 años, posteriormente, se fue con su familia a San José para empezar con sus estudios superiores, provincia donde se encuentra actualmente.

Es importante, señalar que la percepción del ambiente poco después de la Guerra Civil no es similar a la de nuestro otro entrevistado, posiblemente por las diferencias geográficas y por pertenecer a una familia comunista, en un momento de "guerra psicológica", concepto tomado de Alfonso González (2005, p. 20), el cual tiene como propósito explicar cómo se fortalecen ideales homogéneos entre la población en general, manifestándose en contra con reacciones de evitación y distanciamiento respecto a ideas y seguidores adscritos a pensamientos adversos al hegemónico.

A pesar de su edad, Federico Picado nos expresa que hay una continua falta de la figura paterna que ha estado presente durante gran parte de su vida, apareciendo espacios cargados de añoranzas sobre juegos en la playa o salidas familiares a la iglesia, por consiguiente, estamos frente a un indicio 
de melancolía como reacción frente a la pérdida con reproches, asimismo, es distinto a un duelo, pues las características que encontramos en el testimonio con respecto a la violencia de carácter espacial y temporal, son de orden traumático, del duelo no resuelto, y del espectro del padre muerto, que vuelve y revuelve porque no se le pudo decir adiós correctamente.

Es llamativo, cómo nuestro entrevistado (F. Picado, comunicación personal, junio 20,2015) piensa que su padre “...perteneció a una familia de personas que vivieron muchos años, y que entonces por qué...no podría estar vivo hoy...", afirmando que trata "...de sacar la mejor enseñanza...", sin dejarse formar en su interior, alguna "...fuerza de odio ni de venganza si quiera..." gracias a "...los antecedentes que...tenía de formación cristiana".

\section{Conclusiones}

\section{La Guerra del 48:}

El fútbol como puerta de entrada a luchar en la Guerra Civil, siendo la manera en que se unieron algunos jóvenes combatientes al Ejército de Liberación Nacional:

“...estábamos muy contentos de ir a ella, porque el equipo de futbol que eran todos jóvenes estábamos metidos con un rifle, está bien, es una aventura, no queda mucho en ese sentido, pero sí algunas cosas que recuerdo...". (L. Chavarría, comunicación personal, mayo 29, 2015)

La aparición de un caudillo, José Figueres Ferrer:

“... yo recuerdo cuando una vez nos estaba ametrallando un avión en San Isidro del General, en la plaza y Don Pepe cogió una ametralladora de esas que se sacan, es decir, del trípode, se la puso aquí y... "papapapapa" (señala su pecho), comenzó a dispararle al avión. Una gran imagen y por mucho rato una gran imagen...". (L. Chavarría, comunicación personal, mayo 29, 2015)

El avión como arma psicológica:

“...de la guerra el momento que guardo con mayor lucidez es, cuando llegaba el avión y había que dispararle a ese hijueputa avión. El avión es un arma psicológica, que asusta a la gente...". (L. Chavarría, comunicación personal, mayo 29, 2015) 
Una visión de un civil de la guerra que se aparta a la de un excombatiente:

“...fueron cambiando muchas cosas....los mismos dias que...el Ejército de Liberación Nacional...tomaron Limón un domingo en la mañana...y y desde ese momento, se veían los camiones, los soldados andando con toda la vestimenta, con sus cascos, con sus ropas, con sus fajas llenas de tiros con pistolas a la izquierda y a la derecha, con ametralladoras en un hombro y en el otro hombro y...sus botas, entrando a las casas empujando las puertas de las casas, pasaban casa por casa registrando closets... levantado cobijas y las camas, y viendo debajo de las camas...buscando armas, buscando enemigos, gente a quien meter presa y...y a desde ese entonces, empezó Limón... como otro ambiente, se empezaron a ver ya familias divididas". (F. Picado, comunicación personal, junio 20, 2015)

\section{Posguerra y consecuencias de una revolución:}

Federico Picado, nos brinda un excelente relato con respecto a cómo se vivía la persecución a los opositores del gobierno durante la posguerra:

"cuando mi padre fue fusilado por su color político, porque no compaginaba o eran diferentes sus pensamientos a los del gobierno de entonces. Y como él era un líder politico entonces lo, lo tomaban preso muy a menudo y tenían que soltarlo casi que inmediatamente o al día siguiente, porque ya sabíamos nosotros, sobre todo mi mamá como agenciárselas para presentar un recurso de habeas corpus" (F. Picado, comunicación personal, junio 20, 2015)

Para Lisímaco Chavería la Posguerra se vivió distinta:

“...los cincuentas y los sesentas eran años de auge. Sí, salarios altos con las leyes que dejó Calderón Guardia...y comida en todos los rincones de Costa Rica con las leyes que metió José Figueres". (L. Chavarría, comunicación personal, mayo 29, 2015)

Empero, la consecuencia de las persecuciones a bandos opositores se cobraron la vida de un padre amoroso:

"...yo me entere de la muerte de mi padre cuando me topé con su ataúd que estaba ya listo para ser llevado al cementerio... y me dolió mucho que tenía...quebrado el hueso de la frente hundido y... 
yo le pregunte a mi tío que era eso y no me pudo decir, después...me di cuenta qué es que en el trayecto del cuartel hasta el lugar donde los asesinaron, los iban maltratando, los iban golpeando, le iban diciendo las peores cosas que podian sobre su madre y sobre todo, sobre quienes eran ellos. $Y$ en algún momento parece...que usaban la culata de los rifles para maltratarlos, golpearlos, entonces, aparentemente un culatazo fue el que le produjo esa herida a mi padre en la frente, esa fue la forma en que me di cuenta de la muerte de él...". (F. Picado, comunicación personal, junio 20, 2015).

Finalmente, a través de las páginas de esta investigación se revela contantemente la riqueza teórico-metodológica aplicada para reconstruir la oralidad de un evento histórico, en este caso, con respecto a los desenlaces de una guerra. Hoy en la Costa Rica del siglo XXI, trabajos como el presente buscan dar cuenta de la memoria viva de las víctimas o involucrados de la Guerra Civil y sus consecuencias, buscando comprender las diversas cosmovisiones que se impactaron a raíz del gobierno de facto de la Junta Fundadora de la Segunda República, reclamándose desde los recuerdos de los afectados un canal para la discusión de las políticas de la memoria con respecto a la responsabilidad del Estado costarricense en sus acciones históricas. Y por tanto, la historia oral se vería como una herramienta útil para develar esa "recuperación", o mejor dicho, construcción de las distintas memorias a propósito de eventos bélicos que todavía están en construcción para dar una visión más inclusiva de la guerra civil de 1948.

\section{Referencias}

Acuña, Víctor Hugo. (2014). Las memorias del Codo del Diablo. Revista Paquidermo. Recuperado de http://www.revistapaquidermo.com/ archives/11199

Acuña, Víctor Hugo. (1989). Fuentes orales e historia obrera: el caso de los zapateros en Costa Rica. Secuencia. Revista Americana de Ciencias Sociales, núm. 13.

Acuña, Víctor Hugo. (1989). La historia oral, las historias de vida y las Ciencias Sociales. En Fonseca, Elizabeth (Comp.), Historia: teoría y métodos. San José, EDUCA.

Bustillo, Josefina Cuesta. (1998). Memoria e Historia. Un Estado de la Cuestión. Ayer, núm. 32. 
Chavarría, Lisímaco. (2014). Las dos batallas en San Isidro del General: relatos de la revolución y de otros aconteceres. San José: Editorial Industrias Marino.

Favret-Saada, Jeanne. (1991). Sale histoire. Gradhiva, núm. 10.

González Ortega, Alfonso. (2005). Mujeres y hombres de la posguerra costarricense (1950-1960). San José: Editorial de la Universidad de Costa Rica.

Hobsbawm, Eric. La era del Imperio, 1875-1914 (1989). Barcelona: Labor.

Isabel Gamboa Barboza. (2010). Los recuerdos quebrados del 48. Boletín AFEHC, núm. 44. Recuperado de: http://afehc-historia-centroamericana.org/index.php?action=fi_aff\&id $=2368$

Mercedes Muñoz (Ed.). (2003). Niñas y niños del 48 escriben. San José: Editorial Universidad de Costa Rica.

Puyana, Yolanda y Barreto, Juanita. (1994). La historia de vida: recurso en la investigación cualitativa. Reflexiones metodológicas. Maguere, 9(10).

Thompson, Paul. (1978 ). The Voice of the Past: Oral History. Inglaterra: Oxford University Press.

Zapparoli, Mayra. (2003). Los niños y niñas del 48. Herencia, 15(2). 